\title{
SOUTHWEST JOURNAL of PULMONARY $\sigma$ CRITICAL CARE
}

Journal of the Arizona, New Mexico, Colorado and California Thoracic Societies www.swipcc.com

\section{Medical Image of the Month: Superior Vena Cava Syndrome}

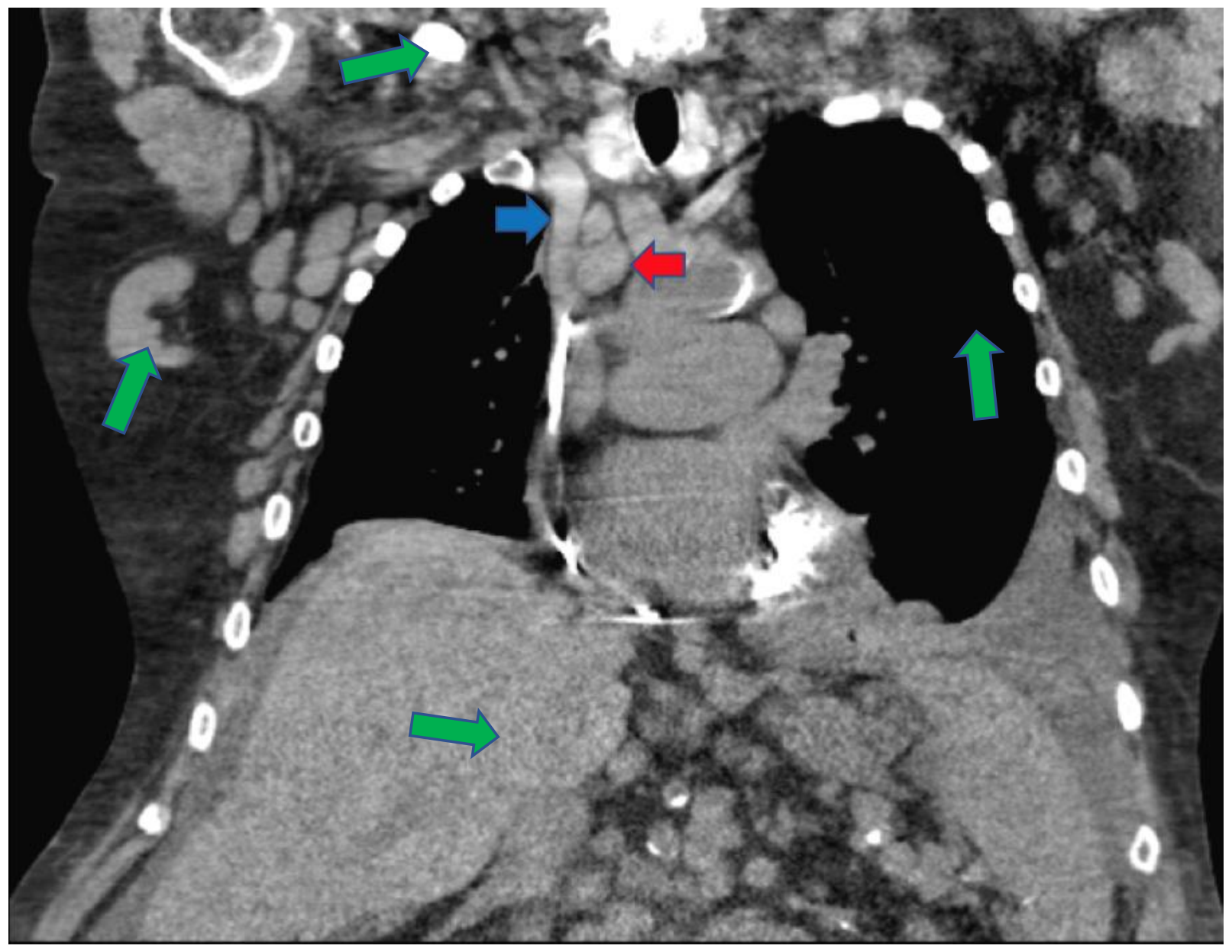

Figure 1. Large mediastinal lymph nodes (red arrow) causing compression of the superior vena cava (blue arrow). Numerous enlarged lymph nodes can also be seen in the axillary, cervical, and upper abdominal regions (green arrows).

\section{History}

A 74-year- old man with a history of diastolic heart failure, chronic kidney disease (CKD), and chronic lymphocytic leukemia (CLL) presented with a complaint of dyspnea. He has had several hospitalizations in the last year for heart failure exacerbation and his home bumetanide was recently increased 
from twice to three times daily due to persistently increasing weight. His CLL was diagnosed two years prior and treatment was stopped three months ago due to side effects. In the emergency department he reported three weeks of worsening dyspnea especially when lying flat, as well as increased swelling in his legs, abdomen, arms, and face. His weight was up to $277 \mathrm{lbs}$ from $238 \mathrm{lbs}$ the month before. His diuretics were transitioned to IV, but over the next few days he remained clinically volume overloaded. A noncontrast chest CT was obtained to help evaluate his ongoing respiratory distress (Figure 1). It demonstrated innumerable lymph nodes involving the cervical, axillary, mediastinal, and upper abdominal regions, which had significantly increased in size and number from prior exam several months before. The CT also showed several particularly bulky lymph nodes which appeared to be compressing the superior vena cava.

\section{Discussion}

The superior vena cava (SVC) is responsible for about one-third of the venous return to the heart. Because of its thin walls relative to arterial vasculature, it is susceptible to compression from adjacent structures which may subsequently impair venous return to the heart, a process known as SVC syndrome. Intrathoracic malignancy is responsible for $60-85 \%$ of cases of SVC syndrome, and common symptoms include facial or neck swelling, swelling of the arms, and dyspnea (1). In this case, the patient's apparent resistance to diuresis was felt to be partially secondary to SVC syndrome. In stable patients, contrast-enhanced CT is the preferred imaging modality if SVC syndrome is suspected, which can define the extent of SVC blockage. Duplex ultrasound may be used first to exclude thrombus. In this patient with acute kidney injury on CKD it was decided to forgo the contrast study to avoid further kidney damage. Management of SVC syndrome depends on severity, with emergent treatment focused on maintaining the airway and endovenous recanalization. Definitive treatment is directed at the underlying cause (2).

After about a week of aggressive IV diuresis, the patient's breathing and volume status improved and he was transitioned back to oral diuretics. He was discharged home with plans for hospice.

Matthew R. Borchart MD, Daniel Yu MD, and Indrajit Nandi MD

University of Arizona College of Medicine, Phoenix

Phoenix, AZ USA

\section{References}

1. Rice TW, Rodriguez RM, Light RW. The superior vena cava syndrome: clinical characteristics and evolving etiology. Medicine (Baltimore). 2006 Jan;85(1):37-42. [CrossRef] [PubMed]

2. Wilson LD, Detterbeck FC, Yahalom J. Clinical practice. Superior vena cava syndrome with malignant causes. N Engl J Med. 2007 May 3;356(18):1862-9. [CrossRef] [PubMed] 